\title{
Growth and erosion of amorphous carbon (a-C:H) films by low-temperature laboratory plasmas containing $\mathrm{H}$ and $\mathrm{N}$ mixtures
}

\author{
Thomas Schwarz-Selinger, Christian Hopf, Chao Sun, Wolfgang Jacob* \\ Max-Planck-Institut für Plasmaphysik, EURATOM Association, Boltzmannstr. 2, 85748 Garching, Germany
}

\begin{abstract}
Growth and erosion of amorphous hydrogenated carbon $(\mathrm{a}-\mathrm{C}: \mathrm{H})$ films from nitrogen-containing gas mixtures was studied in an electron-cyclotron-resonance low-temperature plasma. Deposition and erosion rates were measured as a function of nitrogen admixture and ion energy. At low energy, $\mathrm{N}_{2}$ addition to methane plasmas causes a reduction of the deposition rates that does not exceed significantly the expected reduction due to dilution. At higher ion energies the deposition rate reduces further and finally switches to net erosion. Erosion of a-C:H films in $\mathrm{N}_{2} / \mathrm{H}_{2}$ mixtures is much more efficient than in pure $\mathrm{H}_{2}$ and $\mathrm{N}_{2}$. The erosion rate drops with increasing $\mathrm{N}_{2}$ admixture almost proportional to the total ion flux if the substrate is at floating potential. For higher ion energies the erosion rate dramatically increases and shows a clear maximum at around $25 \% \mathrm{~N}_{2}$ flow ratio.
\end{abstract}

PACS numbers: 52.77.Bn, 52.77.Dq, 81.05.Uw, 81.15.Jj

JNM keywords: Erosion, First Wall Materials, Plasma Materials Interaction, Redeposition, Surface Effects.

PSI-17 keywords: Amorphous Films (a-C:D), Chemical erosion (Chemical Sputtering) Co-deposition, Erosion \& Deposition (Redeposition, Erosion, Deposition), Ion-Surface Interaction

Published in Journal of Nuclear Materials: Received May 2006

Accepted for publication November 08, 2006

Published June 15, 2007

Journal of Nuclear Materials 363-365 (2007) 174-178

doi: 10.1016/j.jnucmat.2007.01.005

\section{INTRODUCTION}

Redeposition of carbon together with hydrogen isotopes is a major concern for the operation of ITER because it will contribute significantly to tritium retention. It was suggested to reduce redeposition in remote areas by adding nitrogen to the divertor [1]. Preliminary laboratory experiments on plasmaassisted deposition of amorphous hydrocarbon $(\mathrm{a}-\mathrm{C}: \mathrm{H})$ films in rf and dc low-pressure plasmas by Mutsukura [2], Vandentop et al. [3], and Tabares et al. [1] have reported on considerable reduction of film growth from methane plasmas if $\mathrm{NO}$ or $\mathrm{N}_{2}$ are admixed to the gas phase. It has been hypothesized that this is due to the scavenging effect of $\mathrm{N}_{2}$ and $\mathrm{NO}$ $[1,4-6]$. Scavenger particles are supposed to transform reactive species into non-reactive ones, thereby reducing the deposition rate. If this idea holds true and can be transferred to the divertor plasma it could mitigate the problem of hydrocarbon redeposition in tokamak experiments and eventually in ITER. The scavenger effect, which was originally invented in photochemistry [7], is, however, only applicable if a limited number of reaction channels exist for the atom or molecule of interest. In the complex environment of a divertor or lowtemperature plasma, where energetic ions, electrons, metastables, and radicals are present the interpretation of experimental observations on the basis of a simplified scavenger effect is insufficient. In addition, presently no proof of any scavenger effect in low-temperature plasma deposition of a-C:H films can be found in literature. This does not necessarily mean that a scavenger effect does not exist, but the tools and methods

*Email address: Wolfgang.Jacob@ipp.mpg.de to investigate it were not specific enough to allow any sound conclusion.

Because of the fact that net deposition of a-C:H films is always a competition between gross deposition and simultaneously occurring erosion [8-11] it is not sufficient to detect a reduction of the net deposition rate as a function of, e.g., $\mathrm{N}_{2}$ or NO admixture to demonstrate the scavenger effect unambiguously. The net deposition rate can also be reduced by enhanced erosion. E.g. chemical sputtering could account for the observed reduction of the net deposition rates. There are many hints in the literature that chemical sputtering plays an important role in the interaction of nitrogen containing hydrocarbon plasmas with a-C:H surfaces [12-17].

\section{EXPERIMENTAL}

Experiments described here were carried out in a remote electron-cyclotron-resonance (ECR) low-temperature laboratory plasma where the energy of the ions hitting the substrate can be varied by application of an additional substrate bias voltage. Details of the ECR setup PLAQ can be found in Ref. [18]. Important to note is that particle production in the bulk plasma is decoupled from the applied experimental conditions at the substrate such as surface temperature and the energy of the impinging ions. To achieve this the plasma is confined in a metallic cage inside the vacuum chamber with solid bottom plate with a central hole $5 \mathrm{~cm}$ in diameter from where a diverging plasma beam is extracted and impinges perpendicularly on the substrate electrode. The substrate electrode is in a distance of $10 \mathrm{~cm}$ from this bottom plate. Because particle generation in the ECR plasma is not affected by the substrate conditions we can differentiate between effects stemming from surface 
effects and effects in the plasma volume.

Ion currents to the substrate were measured using a retarding field analyzer (RFA) at the substrate position [19]. For these measurements the substrate electrode is replaced by the RFA setup. Measurements at floating potential show a mean ion energy of about 4 to $8 \mathrm{eV}$ and an ion flux in the range of some $10^{15} \mathrm{~cm}^{-2} \mathrm{~s}^{-1}$. The potential at the substrate electrode is increased by applying an rf bias at $13.56 \mathrm{MHz}$ to achieve a dc self-bias of up to $-200 \mathrm{~V}$.

PLAQ is equipped with in-situ ellipsometry operating at $632 \mathrm{~nm}$ to measure erosion and deposition rates at the electrically isolated substrate electrode in real time. Single crystalline silicon pieces ( 25 by $25 \mathrm{~mm}^{2}$ ) are used as substrates. A differentially pumped quadrupole mass spectrometer is attached to the chamber in a remote position to investigate the composition of the stable species in the gas phase.

The gas mixtures given in this article are always the ratios of the applied gas flows. The actual gas flows were adjusted such that they resulted in a pressure of $1.0 \mathrm{~Pa}$ before plasma ignition for maximum pumping speed of our setup. This required, e.g., for a pure nitrogen plasma a gas flow of $95 \mathrm{sccm}$ and for pure hydrogen plasmas a gas flow of $38 \mathrm{sccm}$. This difference in gas flow is due to the different pumping speed for hydrogen as compared to nitrogen of the standard turbomolecular pump used [20]. Because of that the actual partial pressures in the chamber are not given by the gas flow ratios.

\section{RESULTS}

\section{A. Deposition of a-C:H:N in $\mathrm{CH}_{4} / \mathrm{N}_{2}$ and $\mathrm{C}_{2} \mathrm{H}_{2} / \mathrm{N}_{2}$ mixtures}

Preliminary growth experiments in the PLAQ device were conducted a few years ago with methane $\left(\mathrm{CH}_{4}\right)$ and acetylene $\left(\mathrm{C}_{2} \mathrm{H}_{2}\right)$ as precursor gases for different $\mathrm{N}_{2}$ admixtures [21]. One aim of these preliminary experiments was to incorporate nitrogen into a-C:H in order to improve hardness and reduce stress. The growth rates from those experiments are depicted in Fig. 1. In contrast to all other measurements shown in this article, the pressure was $0.3 \mathrm{~Pa}$ and microwave power was $50 \mathrm{~W}$. The substrate was kept at floating potential. Ion energy distributions for these conditions were not measured, but we assume that they are slightly higher than those reported in Sect. II due to the lower pressure. One can clearly see that for $\mathrm{CH}_{4}$ as well as for $\mathrm{C}_{2} \mathrm{H}_{2}$ the growth rate is reduced with increasing $\mathrm{N}_{2}$ addition as stated in the literature [1]. However, the measured decrease does not differ significantly from the reduction that would be expected from the simple dilution of the precursor gas. For increased ion energies no films could be grown on the silicon substrates. As mentioned before interpretation of deposition rates in nitrogen containing low-temperature plasmas is not simple and straightforward. An important process that has not been adequately taken into consideration in the past is chemical sputtering which leads to a reduction of the measured net deposition rates. To elucidate the relative importance of erosion in the net deposition of a-C:H:N films we first studied the etching of a-C:H films in $\mathrm{H}_{2} / \mathrm{N}_{2}$ gas mixtures. This process is of importance because

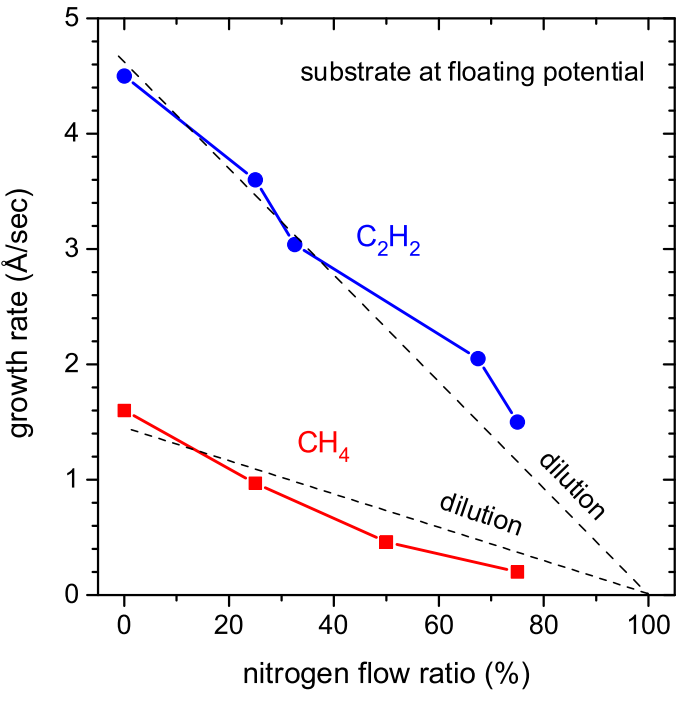

FIG. 1: Deposition rates of a-C:H films as a function of nitrogen admixture for methane and acetylene discharges. The lines are only guides to the eye. The dotted lines illustrate the decay as expected from dilution of the precursor gas.

hydrogen is a dominant reaction product in methane plasmas and contributes strongly to the particle flux to the surface.

\section{B. Substrate erosion with $\mathbf{H}_{2} / \mathbf{N}_{2}$ plasmas}

The erosion of a-C:H films was investigated as a function of nitrogen admixture and applied substrate bias voltage. Results are depicted in Fig. 2. The experiments were conducted in the following way: First, a-C:H films were deposited from a pure methane discharge with the substrate at floating potential resulting in soft, polymer-like films with a refractive index $\hat{n}=1.6-i 0.001$ and a film thickness of around $310 \pm 10 \mathrm{~nm}$. After deposition the sample was covered with a shutter and the chamber was cleaned in an oxygen plasma. The cleanness of the chamber was checked by mass spectrometry. After cleaning the chamber, the intended gas mixture was introduced, the shutter opened, and the plasma started. This procedure warrants that the chamber is always in the same initial state at the beginning of an erosion experiment. In the following this is termed clean chamber conditions. Then the erosion rates were measured for a fixed gas mixture applying different bias voltages from floating potential up to $-200 \mathrm{~V}$.

Increasing the amount of nitrogen with the substrate at at the floating potential causes a monotonic decrease of the erosion rate. This decrease is similar to the reduction of the total ion flux to the substrate surface measured prior to the erosion experiments with the RFA for identical plasma parameters. The erosion yield determined from the measured carbon erosion rate and the measured ion flux remains constant over this parameter range. Therefore, we assume that this decrease is mainly determined by the corresponding decrease of the ion flux. 


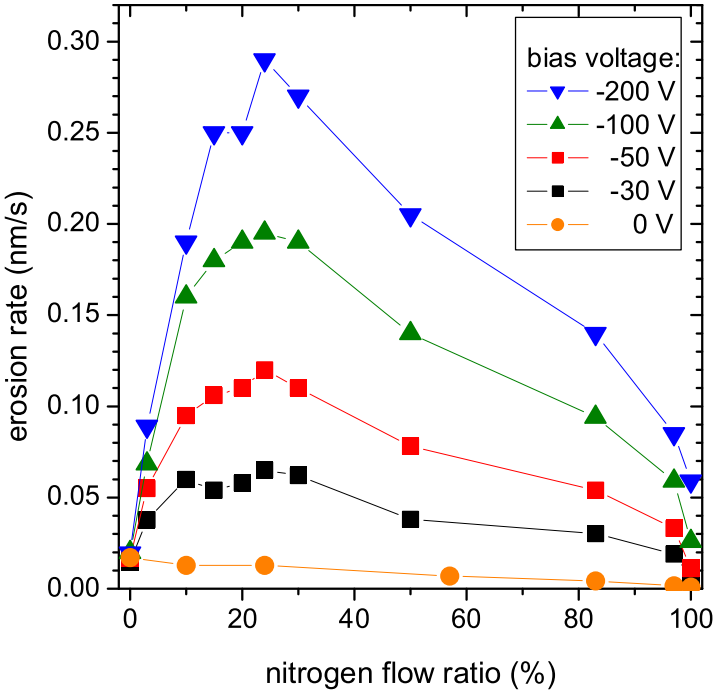

FIG. 2: Erosion rates of dense a-C:H films as a function of nitrogen admixture for different applied substrate bias voltages. The lines are only guides to the eye. The data were determined for clean chamber conditions as described in Sect. III B.

In contrast the erosion rate increases dramatically with increasing nitrogen fraction for the biased case. It shows a maximum around 25\% nitrogen fraction, and then decreases monotonically. Pure hydrogen and pure nitrogen plasmas result in erosion rates which are much lower than the peak values. For pure hydrogen plasmas we find an almost constant erosion rate of about 0.015 to $0.02 \mathrm{~nm} / \mathrm{s}$ for the investigated bias voltages, but for pure nitrogen we measure a significant increase of the rate with increasing bias. The enhanced erosion rate for $\mathrm{H}_{2} / \mathrm{N}_{2}$ mixtures is interpreted as chemical sputtering due to the synergistic interaction of $\mathrm{H}$ and $\mathrm{N}$ at the surface $[10,22]$.

\section{Chamber wall erosion with $\mathrm{H}_{2} / \mathbf{N}_{2}$ plasmas}

Most of the experiments in PLAQ were additionally monitored by mass spectrometry. It turned out that during such an erosion experiment, no significant mass signals from erosion products of the sample could be detected. To get some information on erosion products in the different gas mixtures we performed the following set of experiments: First, the chamber was thoroughly cleaned in an oxygen plasma. Then, a methane plasma with the same parameters as for a-C:H film deposition was run for $12 \mathrm{~min}$. This results in a deposition of an a-C:H film of about $100 \mathrm{~nm}$ on the substrate and an inhomogeneous deposition of $\mathrm{a}-\mathrm{C}: \mathrm{H}$ films on all internal surfaces of the vacuum chamber. After deposition, a plasma with the desired gas mixture was started using identical plasma parameters as during the erosion measurements described in the preceding section. In the following this procedure is termed coated chamber conditions. The process was monitored by measuring time traces of up to 16 mass channels with the mass spectrometer. Fig. 3 shows the erosion of a-C:H films

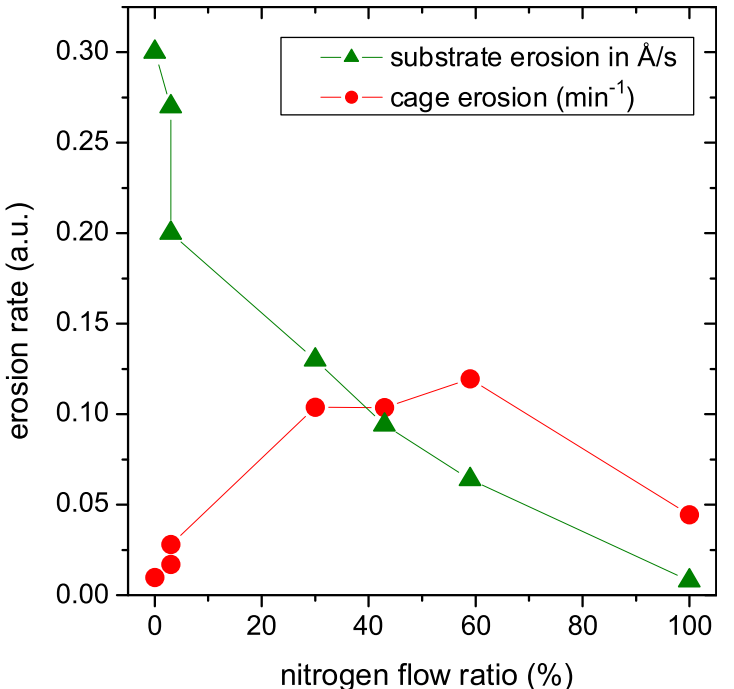

FIG. 3: Erosion rates of a-C:H films measured by ellipsometry at the substrate and cleaning rates of the chamber walls as a function of nitrogen admixture. For the latter we determined the 1/e time for the decrease of the 25 amu mass peak measured by mass spectrometry. The data were determined for coated chamber conditions.

at the substrate at floating potential measured by ellipsometry and the simultaneously measured cleaning rates determined by mass spectrometry. To determine the latter we used the decay of the mass $25 \mathrm{amu}$ signal to $1 / \mathrm{e}$ of its initial value. The erosion rates measured at the substrate show a similar behavior as the data presented in Fig. 2 (floating potential curve) which were measured for clean chamber conditions, but the decrease with increasing nitrogen fraction is stronger than in Fig. 2. The cleaning curve for the chamber walls shows, however, a different behavior. It is similar to the curves measured for biased substrates.

One possible explanation is that the plasma temperature inside the cage is higher than above the substrate. This would lead to a higher sheath potential and consequently to a higher ion energy. The totally different behavior would be explicable if we further assume that we need a certain minimal threshold energy for chemical sputtering. It could be that for the used plasma conditions the ion energy at the substrate is for floating potential just below this threshold and in the cage due to the higher sheath potential just above. For the latter case we would anticipate a behavior similar to the measurements with substrate bias. This point certainly needs more detailed investigations.

\section{Deposition in $\mathrm{CH}_{4} / \mathrm{N}_{2}$ plasmas}

Fig. 4 summarizes erosion rates as a function of bias voltage for pure $\mathrm{H}_{2}$ plasmas and the $\mathrm{N}_{2} / \mathrm{H}_{2}$ plasmas with $30 \% \mathrm{~N}_{2}$ admixture from the preceding sections and compares them with deposition rates measured for pure $\mathrm{CH}_{4}$ plasmas and $\mathrm{CH}_{4} / \mathrm{N}_{2}$ plasmas with $30 \% \mathrm{~N}_{2}$ admixture. For all gases a pressure of 


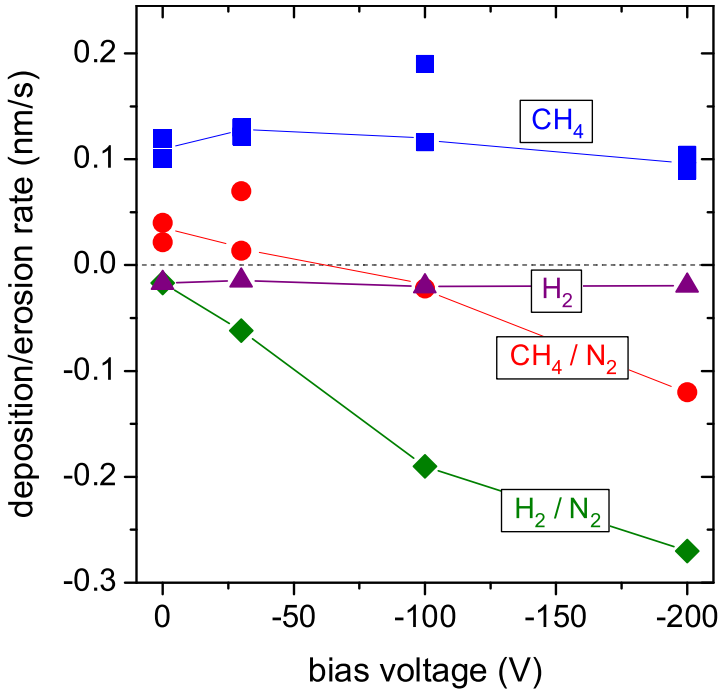

FIG. 4: Deposition and erosion rates in different gas mixtures. All experiments were performed at a pressure of $1.0 \mathrm{~Pa}$ applying a microwave power of $150 \mathrm{~W}$.

1.0 $\mathrm{Pa}$ (before plasma) and a microwave power of $150 \mathrm{~W}$ was applied.

While the deposition rate in a pure $\mathrm{CH}_{4}$ plasmas is nearly constant up to $-200 \mathrm{~V}$ self-bias we find a change over from deposition to erosion for the mixture of $\mathrm{CH}_{4}$ and $\mathrm{N}_{2}$. For low bias voltages $(0$ and $-30 \mathrm{~V})$, the deposition rate is 0.04 and $0.07 \mathrm{~nm} / \mathrm{s}$, respectively. At higher bias $(-100$ and $-200 \mathrm{~V})$, we observe erosion with rates of about 0.02 and $0.12 \mathrm{~nm} / \mathrm{s}$.
This observation cannot be explained by a scavenger effect, because the plasma and in particular the plasma chemical reactions in the gas phase are not changed by application of an additional substrate bias. In addition, the scavenger hypothesis cannot explain erosion. The observed effect has to be dominated by surface reactions. It shows, like the erosion in the $\mathrm{H}_{2} / \mathrm{N}_{2}$ mixture, the clear signature of chemical sputtering.

\section{CONCLUSIONS}

Erosion plays a very significant role in $\mathrm{H}_{2} / \mathrm{N}_{2}$ gas mixtures. In particular at increased ion energies, the admixture of nitrogen leads to a dramatic increase of the erosion rate. For ion energies above $30 \mathrm{eV}$ the erosion rate has a maximum for nitrogen flow ratios around $25 \%$.

In $\mathrm{CH}_{4} / \mathrm{N}_{2}$ mixtures we observe a decrease of the deposition rate with increasing nitrogen fraction. For increased ion energy deposition can turn into net erosion. We attribute this observation to the effect of chemical sputtering and not to a scavenger effect. Because of this increased erosion due to chemical sputtering as found in the data presented, it is not recommended that $\mathrm{N}_{2}$ be added to the divertor plasmas if the plasma-facing components are made of carbon.

A possible experiment to provide a quantitative estimate of the reduction (or enhancement) of the deposition rates in remote areas would be to do cavity investigations in laboratory plasmas [23]. Cavity substrates should be placed in remote areas and the deposition inside should be determined as a function of nitrogen admixture.
[1] F. L. Tabarés, D. Tafalla, I. Tanarro, V. J. Herrero, A. Islyaikin, and C. Maffiotte, Plas. Phys. Contr. Fusion 44, L37 (2002).

[2] N. Mutsukura, Plasma Chem. Plasma Proc. 21, 265 (2001)

[3] G. J. Vandentop, M. Kawasaki, R. M. Nix, I. G. Brown, M. Salmeron, and G. A. Somorjai, Phys. Rev. B 41, 3200 (1990).

[4] F. L. Tabarés and D. Tafalla, Phys. Scripta T103, 47 (2003).

[5] F. L. Tabarés, D. Tafalla, I. Tanarro, V. J. Herrero, and A. M. Islyaikin, Vacuum 73, 161 (2004).

[6] F. L. Tabarés, V. Rohde, and the ASDEX Upgrade Team, Plas. Phys. Contr. Fusion 46, B381 (2004).

[7] H. Okabe, Photochemistry of Small Molecules (Wiley, New York, 1978), 1st ed.

[8] W. Jacob, Thin Solid Films 326, 1 (1998).

[9] A. von Keudell and W. Jacob, J. Appl. Phys. 79, 1092 (1996).

[10] C. Hopf, W. Jacob, and A. von Keudell, J. Appl. Phys. 97 094904 (2005).

[11] W. Jacob, J. Nucl. Mater. 337-339, 839 (2005).

[12] J. Hong and G. Turban, Diamond Relat. Mater. 8, 572 (1999).

[13] J. Hong, A. Granier, A. Goullet, and G. Turban, Diamond Relat. Mater. 9, 573 (2000).
[14] P. Hammer and W. Gissler, Diamond Relat. Mater. 5, 1152 (1996).

[15] N. Hellgren, M. P. Johansson, E. Broitman, P. Sandström, L. Hultman, and J.-E. Sundgren, Thin Solid Films 382, 146 (2001).

[16] N. A. Morrison, S. E. Rodil, J. Robertson, and W. I. Milne, J. Appl. Phys. 89, 5754 (2001).

[17] C. Spaeth, U. Kreissig, and F. Richter, Thin Solid Films 355356, 64 (1999).

[18] B. Landkammer, A. von Keudell, and W. Jacob, J. Nucl. Mater. 264, 48 (1999).

[19] P. Pecher, Ph.D. thesis, Universität Bayreuth (1997).

[20] B. Plöckl and T. Schwarz-Selinger (2005), unpublished.

[21] O. Durand-Drouhin, T. Schwarz-Selinger, and W. Jacob (2000), unpublished.

[22] W. Jacob, C. Hopf, and M. Schlüter, Appl. Phys. Lett 876, 204103 (2005).

[23] C. Hopf, T. Schwarz-Selinger, W. Jacob, and A. von Keudell, J. Appl. Phys. 87, 2719 (2000). 